\title{
Clinical Usefulness of the VS Classification System Using Magnifying Endoscopy with Blue Laser Imaging for Early Gastric Cancer
}

\author{
Yoshikazu Yoshifuku, ${ }^{1}$ Yoji Sanomura, ${ }^{2}$ Shiro Oka, ${ }^{1}$ Kazutaka Kuroki, ${ }^{1}$ Mio Kurihara, \\ Takeshi Mizumoto, ${ }^{1}$ Yuji Urabe, ${ }^{1}$ Toru Hiyama, ${ }^{3}$ Shinji Tanaka, ${ }^{2}$ and Kazuaki Chayama ${ }^{1}$ \\ ${ }^{1}$ Department of Gastroenterology and Metabolism, Graduate School of Biomedical Sciences, Hiroshima University, Hiroshima, Japan \\ ${ }^{2}$ Department of Endoscopy, Hiroshima University Hospital, Hiroshima, Japan \\ ${ }^{3}$ Health Service Center, Hiroshima University, Higashihiroshima, Japan
}

Correspondence should be addressed to Yoji Sanomura; y-sanomura@hiroshima-u.ac.jp

Received 16 February 2017; Accepted 5 April 2017; Published 17 May 2017

Academic Editor: Tatsuya Toyokawa

Copyright (C) 2017 Yoshikazu Yoshifuku et al. This is an open access article distributed under the Creative Commons Attribution License, which permits unrestricted use, distribution, and reproduction in any medium, provided the original work is properly cited.

Background. Blue laser imaging (BLI) enables the acquisition of more information from tumors' surfaces compared with white light imaging. Few reports confirm the validity of magnifying endoscopy (ME) with BLI (ME-BLI) for early gastric cancer (EGC). We aimed to assess the detailed endoscopic findings from EGCs using ME-BLI. Methods. We enrolled 386 consecutive patients with 417 EGCs that were diagnosed using ME-BLI and resected by endoscopic submucosal dissection. Using the VS classification system, three highly experienced endoscopists (HEEs) and three less experienced endoscopists (LEEs) evaluated the demarcation line (DL), microsurface pattern (MSP), and microvascular pattern (MVP) within the endoscopic images of EGCs obtained using ME-BLI, assigning high-confidence (HC) or low-confidence (LC) levels. We investigated the clinicopathological features associated with each confidence level. Results. The HEEs' evaluations determined the presence of DL in 99\%, irregular MSP in 96\%, and irregular MVP in 96\%, and the LEEs' evaluations determined the presence of DL in 98\%, irregular MSP in 95\%, and irregular MVP in $95 \%$ of the EGCs. When DL was present, HC levels in the Helicobacter pylori- (H. pylori-) eradicated group and noneradicated group were evident in $65 \%$ and $89 \%$, a difference that was significant $(p<0.001)$. Conclusions. In the diagnosis of EGC with ME-BLI, the VS classification system with ME-NBI can be applied, but identifying the DL after $H$. pylori was difficult.

\section{Introduction}

Gastric cancer is one of the most common cancers, and it is the second leading cause of cancer deaths worldwide [1]. Endoscopic submucosal dissection (ESD) is performed worldwide to treat early gastric cancer (EGC). In Japan, ESD for EGC is performed in accordance with the Japanese gastric cancer treatment guidelines [2], and it can attain higher en bloc resection and curative resection rates and good prognoses, even for large or ulcerated lesions [3-7]. To perform ESD, it is important to accurately diagnose the gastric cancer at an early stage. However, it is difficult to diagnose EGCs using white light imaging (WLI) only because
EGCs can sometimes appear to be assimilated within the surrounding mucosa. Although chromoendoscopy using indigo carmine dye or an acetic acid-indigo carmine dye mixture has been reported to be useful for EGC detection [8-13], these procedures take time and effort.

In recent years, many image-enhanced endoscopy (IEE) techniques, including narrow band imaging (NBI), flexible spectral imaging color enhancement (FICE), and blue laser imaging (BLI), have been developed to improve the visualization of the vascular and surface patterns within the surface of the mucosa, and, therefore, diagnoses. Several reports describe the value of IEE for gastric tumors [14-17]. IEE is widely used because of its simplicity. Indeed, it only requires 
TABle 1: Clinical characteristics of the patients $(n=386)$ and the early gastric cancers $(n=417)$.

\begin{tabular}{lc}
\hline Characteristic & \\
\hline Sex ratio (male/female) & $280 / 106$ \\
Age, years, mean (SD) & $70.3(10.4)$ \\
Tumor location, $n$ (\%) & $74(18)$ \\
$\quad$ Upper & $138(33)$ \\
Middle & $205(49)$ \\
Lower & \\
Macroscopic type, $n(\%)$ & $142(34)$ \\
0-I, 0-IIa & $275(66)$ \\
0-IIb, 0-IIc & $18.6(13.2)$ \\
Tumor size, mm, mean (SD) & \\
Histological type, $n(\%)$ & $383(92)$ \\
Differentiated & $34(8)$ \\
Undifferentiated & \\
Depth, $n$ (\%) & $377(90)$ \\
T1a & $40(10)$ \\
T1b & \\
Helicobacter pylori & $79(19)$ \\
Eradicated, $n(\%)$ & $338(81)$ \\
Noneradicated, $n(\%)$ & \\
\hline
\end{tabular}

SD: standard deviation.

a button to be pushed, and unlike chromoendoscopy, a dye solution is not needed. Furthermore, more detailed information about EGCs can be obtained when magnifying endoscopy (ME) is combined with IEE. NBI is the form of IEE most used in Japan. Yao et al. [14] described the VS classification system, which is an approach that facilitates the diagnosis of EGC using ME and NBI, and they emphasized three factors to consider during the diagnosis of EGC, namely, the presence of a demarcation line (DL), an irregular microvascular pattern (MVP), and an irregular microsurface pattern (MSP). The criteria that define a diagnosis of EGC are either the presence of an irregular MVP with a DL or the presence of an irregular MSP with a DL.

BLI is an IEE technique that uses the LASEREO system, which is a laser-based endoscopy system. Only one publication suggests that ME-BLI may be useful for diagnosing EGC [17], and there are no reports that detail the endoscopic findings attained using ME-BLI. In the present study, we aimed to assess the validity of the VS classification system using ME-BLI for EGC.

\section{Patients and Methods}

2.1. Patients. We enrolled 386 consecutive patients who had 417 EGCs that were diagnosed using BLI and who had undergone ESD resections at Hiroshima University Hospital between August 2011 and March 2016. Table 1 presents the characteristics of the study participants and their tumors. All of the patients provided written informed consent to undergo ESD. The study was approved by Hiroshima
University's Institutional Review Board and its Ethics Committee. In addition, this study was performed in accordance with the principles of the Declaration of Helsinki.

2.2. Imaging Techniques Using the BLI System. The LASEREO endoscopy system (Fujifilm Co., Tokyo, Japan) consists of an LL-4450 light source, a VP-4450HD video processor, and any of a special series of scopes. The LL- 4450 light source provides illumination through two different lasers that have mean wavelengths of 410 (standard deviation $(\mathrm{SD})=10) \mathrm{nm}$ and $450(\mathrm{SD}=10) \mathrm{nm}$. The $450 \mathrm{~nm}$ wavelength laser excites the white light phosphor and produces fluorescent light for standard observations, while the $410 \mathrm{~nm}$ wavelength laser is for BLI, which functions as narrow band imaging. The lighting setup offers three observation modes, namely, the BLI mode, the BLI-bright mode, and the white light mode, which can be selected by adjusting the intensity of the two lasers. The BLI mode is a combination of a strong $410 \mathrm{~nm}$ laser light, a weak $450 \mathrm{~nm}$ laser light, and a fluorescent light. The BLI-bright mode is a combination of a strong $410 \mathrm{~nm}$ laser light, a $450 \mathrm{~nm}$ laser that is stronger than the $450 \mathrm{~nm}$ laser used for the BLI mode, and a fluorescent light. The white light mode is a combination of a weak $410 \mathrm{~nm}$ laser light, a strong $450 \mathrm{~nm}$ laser light, and a fluorescent light.

We used BLI endoscopes (EG-L600ZW and EGL590ZW; Fujifilm Co., Tokyo, Japan), light sources (LASEREO LL-4450; Fujifilm Co., Tokyo, Japan), and video processors (Advancia HD VP-4450HD; Fujifilm Co., Tokyo, Japan). For the BLI mode, the structure enhancement function and the color mode were set at the B8 level and level 1.

2.3. Image Evaluation. Three highly experienced endoscopists (HEEs) who had diagnosed $>500$ cases using ME-BLI and three less experienced endoscopists (LEEs) who had diagnosed $\leq 500$ cases using ME-BLI retrospectively participated in the evaluation of the images. The endoscopic images of the EGCs were obtained using ME-BLI at a magnification of up to $\times 100$, and they were randomly presented to each of the physicians who evaluated the images for the presence or absence of DL and to determine whether the MSPs were regular, irregular, or absent and whether the MVPs were regular, irregular, or absent using the VS classification system (Figure 1). The endoscopists assigned high-confidence (HC) or low-confidence (LC) levels to their evaluations. If individual diagnostic interpretations differed, the three endoscopists discussed the case until consensus was reached. We investigated the clinicopathological features associated with each confidence level for the EGCs that showed the presence of DL, irregular MSP, and irregular MVP. We also assessed the interobserver agreements between the two groups of endoscopists.

2.4. Statistical Analysis. The quantitative data are expressed as the means and the SDs or percentages. The differences in the values were analyzed using the chi-square test with Yates' correction or using Student's $t$-test. A value of $p<0.05$ was considered statistically significant. The interobserver agreements were measured using the kappa statistic. 


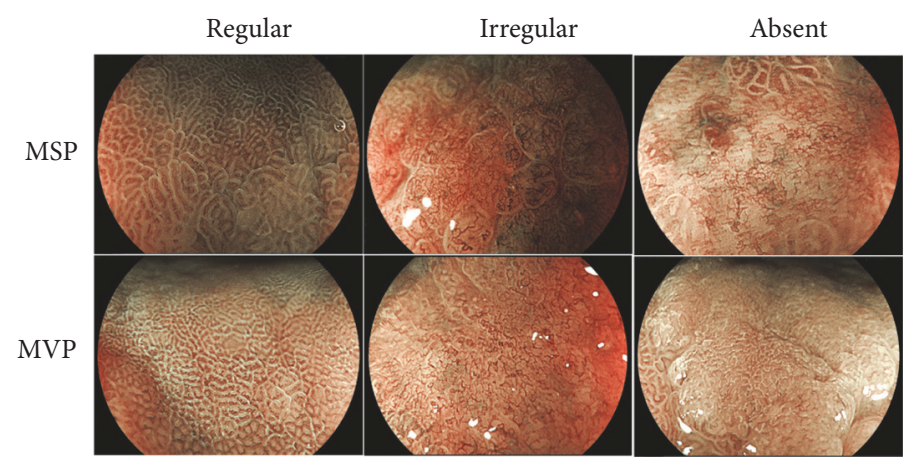

FIgURE 1: Endoscopic images of the VS classification system using ME-BLI.

TABLE 2: ME-BLI findings based on the VS classification system.

\begin{tabular}{|c|c|c|c|c|c|c|c|c|c|}
\hline \multirow[b]{2}{*}{ Endoscopist } & \multirow{2}{*}{$\begin{array}{l}\text { Confidence } \\
\text { level }\end{array}$} & \multicolumn{2}{|c|}{$\mathrm{DL}$} & \multicolumn{3}{|c|}{ MSP } & \multicolumn{3}{|c|}{ MVP } \\
\hline & & $\begin{array}{c}\text { Present, } \\
n(\%)\end{array}$ & $\begin{array}{c}\text { Absent, } \\
n(\%)\end{array}$ & $\begin{array}{c}\text { Regular, } \\
n(\%)\end{array}$ & $\begin{array}{c}\text { Irregular, } \\
n(\%)\end{array}$ & $\begin{array}{c}\text { Absent, } n \\
(\%)\end{array}$ & $\begin{array}{c}\text { Regular, } \\
n(\%)\end{array}$ & $\begin{array}{c}\text { Irregular, } \\
n(\%)\end{array}$ & $\begin{array}{c}\text { Absent, } \\
n(\%)\end{array}$ \\
\hline \multirow{3}{*}{ HEE } & High & $351(85)$ & $0(0)$ & $0(0)$ & $350(84)$ & $6(1)$ & $0(0)$ & $378(91)$ & $11(3)$ \\
\hline & Low & $64(14)$ & $2(1)$ & $6(1)$ & $51(12)$ & $4(1)$ & $2(1)$ & $23(6)$ & $3(1)$ \\
\hline & Total & 415 (99) & $2(1)$ & $6(1)$ & $401(96)$ & $10(2)$ & $2(1)$ & $401(96)$ & $14(3)$ \\
\hline \multirow{3}{*}{ LEE } & High & $342(82)$ & $0(0)$ & $0(0)$ & $346(83)$ & $4(1)$ & $0(0)$ & $360(86)$ & $10(2)$ \\
\hline & Low & $68(16)$ & $7(1)$ & $8(1)$ & $52(12)$ & $7(2)$ & $4(1)$ & $37(9)$ & $6(1)$ \\
\hline & Total & $410(98)$ & $7(1)$ & $8(1)$ & $398(95)$ & $11(3)$ & $4(1)$ & $397(95)$ & $16(4)$ \\
\hline
\end{tabular}

DL: demarcation line; MSP: microsurface pattern; MVP: microvascular pattern; HEE: highly experienced endoscopist: LEE: less experienced endoscopist.

\section{Results}

Table 2 presents the ME-BLI findings based on the VS classification system. The evaluation of the images by the HEEs showed that DL was present in 99\% (415/417) of the EGCs and that the total HC rate was 85\% (351/417). Regular, irregular, and absent MSPs were observed in 1\% (6/417), $96 \%(401 / 417)$, and $2 \%(10 / 417)$ of the EGCs, respectively, and the total $\mathrm{HC}$ rate was $85 \%$ (356/417). Regular, irregular, and absent MVPs were observed in 1\% (2/417), 96\% (401/417), and 1\% (3/417) of the EGCs, respectively, and the total HC rate was 93\% (389/417). The evaluation of the images by the LEEs showed that DL was present in 98\% (410/417) of the EGCs and that the total HC rate was $82 \%$ (342/417). Regular, irregular, and absent MSPs were observed in $2 \%(8 / 417), 95 \%(398 / 417)$, and $3 \%$ (11/417) of the EGCs, respectively, and the total HC rate was $84 \%$ (350/417). Regular, irregular, and absent MVPs were observed in $1 \%(4 / 417), 95 \%$ (397/417), and $4 \%$ (16/417) of the EGCs, respectively, and the total HC rate was $89 \%$ (370/417). There were no significant differences between the HEEs and LEEs with respect to the endoscopic findings.

Table 3 presents the HEEs' confidence levels with respect to the ME-BLI findings relative to the clinicopathological features of the tumors. In the EGCs in which the DL was present, HC levels were evident in 65\% (51/78) and $89 \%$ (300/337) of the Helicobacter pylori- (H. pylori-) eradicated group and the $H$. pylori-noneradicated group, respectively. The $\mathrm{HC}$ rate in the $H$. pylori-noneradicated group was significantly higher than that in the $H$. pylorieradicated group $(p<0.01)$. In EGCs in which the MSP was irregular, the mean tumor sizes with LC and HC levels were $8(\mathrm{SD}=4) \mathrm{mm}$ and $15(\mathrm{SD}=11) \mathrm{mm}$, and there was a significant difference between the LC and HC levels. In EGCs in which the MVP was irregular, mean tumor sizes of LC and HC levels were $8(\mathrm{SD}=5) \mathrm{mm}$ and $14(\mathrm{SD}=10) \mathrm{mm}$, and there was a significant difference between the LC and $\mathrm{HC}$ levels. The interobserver agreements with respect to the DL, MSP, and MVP for the HEEs were $0.78,0.72$, and 0.76 , respectively, and for the LEEs were $0.66,0.61$, and 0.65 , respectively, (Table 4). The interobserver agreements were good-to-satisfactory between the groups.

\section{Discussion}

This study's findings demonstrate that ME-BLI facilitates the acquisition of detailed information about the microvascular and microsurface patterns within the mucosal surfaces of EGCs. Currently, BLI and NBI are used widely as IEE based on narrow band observation function in esophagogastroduodenoscopy. Several publications have described the reliability of ME-NBI at characterizing and delineating EGC $[14,18-$ 21]. The findings from a study by Yamada et al. [18] showed that the sensitivity, specificity, and accuracy of ME-NBI in relation to the diagnosis of EGC were excellent at 95\%, 97\%, and 97\%, respectively. Ezoe et al. [19] showed that the sensitivity, specificity, and accuracy of ME-NBI in relation to the diagnosis of EGC were 95.0\%, 96.8\%, and 96.6\%, respectively. Hence, ME-NBI yielded excellent diagnostic 
TABLE 3: The HEEs' confidence levels in relation to the ME-BLI findings relative to the clinicopathological features of the tumors.

\begin{tabular}{|c|c|c|c|c|c|c|c|c|c|}
\hline \multirow[b]{2}{*}{$\begin{array}{l}\text { Clinicopathological } \\
\text { feature }\end{array}$} & \multicolumn{2}{|c|}{ DL present } & \multirow[b]{2}{*}{$\begin{array}{c}p \\
\text { value }\end{array}$} & \multicolumn{2}{|c|}{ MSP irregular } & \multicolumn{4}{|c|}{ MVP irregular } \\
\hline & $\begin{array}{c}\text { Low } \\
\text { confidence } \\
(n=64)\end{array}$ & $\begin{array}{c}\text { High } \\
\text { confidence } \\
(n=351)\end{array}$ & & $\begin{array}{c}\text { Low } \\
\text { confidence } \\
(n=51)\end{array}$ & $\begin{array}{l}\text { High } \\
\text { confidence } \\
(n=350)\end{array}$ & $p$ value & $\begin{array}{c}\text { Low } \\
\text { confidence } \\
(n=23)\end{array}$ & $\begin{array}{l}\text { High } \\
\text { confidence } \\
(n=378)\end{array}$ & $p$ value \\
\hline \multicolumn{10}{|l|}{ Location, $n(\%)$} \\
\hline Upper & $15(20)$ & $59(80)$ & & $12(17)$ & $60(83)$ & & $5(7)$ & $66(93)$ & \\
\hline Middle & $21(15)$ & $117(85)$ & NS & $18(12)$ & $128(88)$ & NS & $7(5)$ & $126(95)$ & NS \\
\hline Lower & $28(14)$ & $175(86)$ & & $21(11)$ & $162(89)$ & & $11(6)$ & $186(94)$ & \\
\hline \multicolumn{10}{|l|}{ Macroscopic type, $n(\%)$} \\
\hline 0-I, 0-IIa & $15(11)$ & $127(89)$ & NS & $14(10)$ & $120(90)$ & NS & $5(4)$ & $132(96)$ & NS \\
\hline 0-IIb, 0-IIc & $49(18)$ & $224(82)$ & & $37(14)$ & $230(86)$ & & $18(7)$ & $246(93)$ & \\
\hline $\begin{array}{l}\text { Tumor size, mm, mean } \\
\text { (SD) }\end{array}$ & $12(9)$ & $15(11)$ & NS & $8(4)$ & $15(11)$ & $<0.01$ & $8(5)$ & $14(10)$ & $<0.05$ \\
\hline \multicolumn{10}{|l|}{ Histological type, $n(\%)$} \\
\hline Differentiated & $59(15)$ & $323(85)$ & NS & $42(11)$ & $329(89)$ & NS & $20(5)$ & 349 (95) & NS \\
\hline Undifferentiated & $5(15)$ & $28(85)$ & & $9(29)$ & $22(71)$ & & $3(9)$ & $29(91)$ & \\
\hline \multicolumn{10}{|l|}{ Depth, $n(\%)$} \\
\hline T1a & $60(16)$ & $315(84)$ & NS & $49(13)$ & $316(87)$ & NS & $21(6)$ & $343(94)$ & NS \\
\hline $\mathrm{T} 1 \mathrm{~b}$ & $4(10)$ & $36(90)$ & & $2(4)$ & $44(96)$ & & $2(5)$ & $35(95)$ & \\
\hline \multicolumn{10}{|l|}{$\begin{array}{l}\text { Helicobacter pylori } \\
\text { eradication, } n(\%)\end{array}$} \\
\hline Eradicated & $27(35)$ & $51(65)$ & $<0.001$ & $15(20)$ & $60(80)$ & NS & $8(11)$ & $68(89)$ & NS \\
\hline Noneradicated & $37(11)$ & $300(89)$ & & $36(11)$ & $290(89)$ & & $15(5)$ & $310(95)$ & \\
\hline
\end{tabular}

SD: standard deviation; DL: demarcation line; MSP: microsurface pattern; MVP: microvascular pattern; NS: not significant.

TABLE 4: Interobserver agreements for the ME-BLI findings based on the VS classification system.

\begin{tabular}{lcc}
\hline \multirow{2}{*}{ ME-BLI finding } & \multicolumn{2}{c}{ Interobserver agreement } \\
& HEE & LEE \\
\hline DL & 0.78 & 0.66 \\
MSP & 0.72 & 0.61 \\
MVP & 0.76 & 0.65 \\
\hline
\end{tabular}

DL: demarcation line; MSP: microsurface pattern; MVP: microvascular pattern; HEE: highly experienced endoscopist; LEE: less experienced endoscopist.

performances with respect to accuracy, sensitivity, and specificity in both of these studies. In addition to obtaining histopathological diagnoses of EGC, it is important to use ESD to assess the lateral extents of EGCs, and ME-NBI has been reported to be very useful at assessing the lateral extents of differentiated-type EGCs [22-25]. However, it is difficult to assess the lateral extents of undifferentiated-type EGCs, even when ME-NBI is used, because of the presence of proliferative zone extensions. Horiuchi et al. [26] reported that $81.6 \%$ of EGCs could be correctly diagnosed based on the DL when ME-NBI was used. However, it seems that this accuracy is not sufficient when the lateral extents of EGCs appropriate for ESD are being assessed, and this represents a diagnostic limitation associated with optical biopsies.

Regarding the diagnostic yield for EGCs when ME-BLI is used, Dohi et al. [17] reported that compared with WLI, ME-
BLI had an improved diagnostic performance. They showed that the sensitivity, specificity, and accuracy of ME-NBI in relation to the diagnosis of EGCs were excellent at 94\%, $92 \%$, and $92 \%$, respectively, and that the diagnostic effectiveness of ME-BLI was similar to that of ME-NBI. However, the study by Dohi et al. [17] is the only one that has investigated the clinical utility of ME-BLI, and there are no publications that have described investigations into DL, MSP, and MVP, which constitute an EGC diagnosis, the confidence levels, the need for proficiency, and the clinicopathological features of EGC using ME-BLI. In this study, the presence of a DL, an irregular MSP, and an irregular MVP was observed in more than $95 \%$ of the EGCs, in both the HEE and LEE groups, and the HC levels observed for all of these characteristics were more than $80 \%$. There were no significant differences between the HEEs and LEEs with respect to the endoscopic findings and the confidence levels, which suggests that most of the EGC diagnoses undertaken using ME-BLI can be performed easily, regardless of the level of the endoscopist's experience.

Some of the EGC diagnoses were difficult even for the HEEs, including small EGCs or EGCs after $H$. pylori eradication. H. pylori infection causes gastric cancer [27-30], and metachronous EGC was reduced to one-third with successful $H$. pylori eradication therapy after ESD for EGC [31]. Several studies' findings show that it is often difficult to diagnose EGCs after $H$. pylori eradication therapy, because of the tumors' indistinct borderlines or lack of obviously cancerous characteristics [32-34]. Furthermore, we reported that, endoscopically, gastric cancers changed to flattened and 
indistinct forms after $H$. pylori eradication and that nonneoplastic epithelium covered the cancerous areas $[35,36]$. The findings from this study showed that the HC rates associated with DL were 65\% in the H. pylori-eradicated group and $89 \%$ in the $H$. pylori-noneradicated group, a difference that was significant. Hence, we should be careful when we perform endoscopic surveillance to ensure that we do not miss EGC or misunderstand the range of the tumor in patients after H. pylori eradication.

This study has some limitations that are described next. First, this study was retrospective and it involved a review of endoscopic images. Hence, it may not reflect the realtime prospective diagnoses that occur during surveillance endoscopy. Second, this study investigated cancerous lesions only. To avoid bias, a prospective study should be performed that enrolls lesions that have not undergone pathological diagnoses. Third, the study was conducted at a single academic center in Japan; hence, the study's data may lack generalizability to gastric cancer treatment centers worldwide.

We conclude that in diagnosis of EGC with ME-BLI, the VS classification system with ME-NBI can be applied. However, it tends to be difficult to identify the DL of EGCs after $H$. pylori eradication.

\section{Ethical Approval}

All procedures followed were in accordance with the ethical standards of the responsible committee on human experimentation (institutional and national) and with the Helsinki Declaration of 1964 and later versions.

\section{Consent}

The authors obtained informed consent to publish from all patients. Informed consent or substitute for it was obtained from all patients for being included in the study.

\section{Disclosure}

All authors disclosed no financial relationship relevant to the publication.

\section{Conflicts of Interest}

The authors declare that they have no competing interests.

\section{Authors' Contributions}

Yoshikazu Yoshifuku designed this study, collected the clinical data, wrote the manuscript, and performed the statistical analyses, with contributions from Yoji Sanomura, Shinji Tanaka, and Kazuaki Chayama. Shiro Oka, Mio Kurihara, Takeshi Mizumoto, Kazutaka Kuroki, Yuji Urabe, and Toru Hiyama participated in the study as physicians who treated and performed follow-up of the patients. All authors read and approved the submission of the final manuscript.

\section{References}

[1] J. Ferlay, H. R. Shin, F. Bray, D. Forman, C. Mathers, and D. M. Parkin, "Estimates of worldwide burden of cancer in 2008: GLOBOCAN 2008," International Journal of Cancer, vol. 127, no. 12, pp. 2893-2917, 2010.

[2] Japanese Gastric Cancer Association, "Japanese gastric cancer treatment guidelines 2010 (ver. 3)," Gastric Cancer, vol. 14, no. 2, pp. 113-123, 2011.

[3] S. Oka, S. Tanaka, I. Kaneko et al., "Advantage of endoscopic submucosal dissection compared with EMR for early gastric cancer," Gastrointestinal Endoscopy, vol. 64, no. 6, pp. 877883,2006

[4] H. Ono, H. Kondo, T. Gotoda et al., "Endoscopic mucosal resection for treatment of early gastric cancer," Gut, vol. 48, no. 2, pp. 225-229, 2001.

[5] T. Gotoda, "Endoscopic resection of early gastric cancer," Gastric Cancer, vol. 10, no. 1, pp. 1-11, 2007.

[6] A. Imagawa, H. Okada, Y. Kawahara et al., "Endoscopic submucosal dissection for early gastric cancer: results and degrees of technical difficulty as well as success," Endoscopy, vol. 38, no. 10, pp. 987-990, 2006.

[7] T. Gotoda, H. Yamamoto, and R. M. Soetikno, "Endoscopic submucosal dissection of early gastric cancer," Journal of Gastroenterology, vol. 41, no. 10, pp. 929-942, 2006.

[8] K. Ida, Y. Hashimoto, S. Takeda, K. Murakami, and K. Kawai, "Endoscopic diagnosis of gastric cancer with dye scattering," The American Journal of Gastroenterology, vol. 63, no. 4, pp. 316-320, 1975.

[9] S. Yoshinaga, T. Gotoda, and I. Oda, "Clinical imaging of early gastric cancers-conventional endoscopy: including chromoendoscopy using indigo carmine," Stomach Intestine, vol. 44, no. 4, pp. 650-662, 2009, [Article in Japanese].

[10] T. Iizuka, D. Kikuchi, S. Hoteya, and N. Yahagi, "The acetic acid + indigocarmine method in the delineation of gastric cancer," Journal of Gastroenterology and Hepatology, vol. 23, no. 9, pp. 1358-1361, 2008.

[11] Y. Sakai, R. Eto, J. Kasanuki et al., "Chromoendoscopy with indigo carmine dye added to acetic acid in the diagnosis of gastric neoplasia: a prospective comparative study," Gastrointestinal Endoscopy, vol. 68, no. 4, pp. 635641, 2008

[12] Y. Kawahara, R. Takenaka, H. Okada et al., "Novel chromoendoscopic method using an acetic acid-indigocarmine mixture for diagnostic accuracy in delineating the margin of early gastric cancers," Digestive Endoscopy, vol. 21, no. 1, pp. 14-19, 2009.

[13] S. Oka, S. Tanaka, N. Numata, Y. Sanomura, and K. Chayama, "Endoscopic diagnosis of early gastric cancer," Nihon Rinsho, vol. 70, no. 10, pp. 1742-1747, 2012, [Article in Japanese].

[14] K. Yao, G. K. Anagnostopoulos, and K. Ragunath, "Magnifying endoscopy for diagnosing and delineating early gastric cancer," Endoscopy, vol. 41, no. 5, pp. 462-467, 2009.

[15] R. Mouri, S. Yoshida, K. Chayama, S. Tanaka, S. Oka, and M. Yoshihara, "Evaluation and validation of computed virtual chromoendoscopy in early gastric cancer," Gastrointestinal Endoscopy, vol. 69, no. 6, pp. 1052-1058, 2009.

[16] H. Osawa, H. Yamamoto, K. Satoh, and K. Sugano, "Diagnosis of extent of early gastric cancer using flexible spectral imaging color enhancement," World Journal of Gastrointestinal Endoscopy, vol. 4, no. 8, pp. 356-361, 2012. 
[17] O. Dohi, N. Yagi, A. Majima et al., "Diagnostic ability of magnifying endoscopy with blue laser imaging for early gastric cancer: a prospective study," Gastric Cancer, vol. 20, no. 2, pp. 297-303, 2017, [Epub ahead of print].

[18] S. Yamada, H. Doyama, K. Yao et al., "An efficient diagnostic strategy for small, depressed early gastric cancer with magnifying narrow-band imaging: a post-hoc analysis of a prospective randomized controlled trial," Gastrointestinal Endoscopy, vol. 79, no. 1, pp. 55-63, 2014.

[19] Y. Ezoe, M. Muto, N. Uedo et al., "Magnifying narrowband imaging is more accurate than conventional white-light imaging in diagnosis of gastric mucosal cancer," Gastroenterology, vol. 141, no. 6, pp. 2017-2025, 2011.

[20] T. Kanesaka, N. Uedo, K. Yao et al., “A significant feature of microvessels in magnifying narrow-band imaging for diagnosis of early gastric cancer," Endoscopy International Open, vol. 3, no. 6, pp. E590-E596, 2015.

[21] K. Uchita, K. Yao, N. Uedo et al., "Highest power magnification with narrow-band imaging is useful for improving diagnostic performance for endoscopic delineation of early gastric cancers," BMC Gastroenterology, vol. 15, no. 1, p. 155, 2015.

[22] T. Nagahama, K. Yao, S. Maki et al., "Usefulness of magnifying endoscopy with narrow-band imaging for determining the horizontal extent of early gastric cancer when there is an unclear margin by chromoendoscopy (with video)," Gastrointestinal Endoscopy, vol. 74, no. 6, pp. 1259-1267, 2011.

[23] S. Kiyotoki, J. Nishikawa, M. Satake et al., "Usefulness of magnifying endoscopy with narrow-band imaging for determining gastric tumor margin," Journal of Gastroenterology and Hepatology, vol. 25, no. 10, pp. 1636-1641, 2010.

[24] K. Nonaka, M. Namoto, H. Kitada et al., "Usefulness of the DL in ME with NBI for determining the expanded area of early-stage differentiated gastric carcinoma," World Journal of Gastrointestinal Endoscopy, vol. 4, no. 8, pp. 362367, 2012.

[25] M. Takeuchi, M. Kobayashi, S. Hashimoto, R. Narisawa, and Y. Aoyagi, "Usefulness of magnifying narrow band imaging for assessing lateral tumor extent of early gastric cancer: a case report," Digestive Endoscopy, vol. 23, Supplement 1, pp. 86-88, 2011.

[26] Y. Horiuchi, J. Fujisaki, N. Yamamoto et al., "Accuracy of diagnostic demarcation of undifferentiated-type early gastric cancers for magnifying endoscopy with narrow-band imaging: endoscopic submucosal dissection cases," Gastric Cancer, vol. 19, no. 2, pp. 515-523, 2016.

[27] D. Forman, D. G. Newell, F. Fullerton et al., "Association between infection with Helicobacter pylori and risk of gastric cancer: evidence from a prospective investigation," $B M J$, vol. 302, no. 6788, pp. 1302-1305, 1991.

[28] J. Parsonnet, G. D. Friedman, D. P. Vandersteen et al., "Helicobacter pylori infection and the risk of gastric carcinoma," The New England Journal of Medicine, vol. 325, no. 16, pp. 1127-1131, 1991.

[29] A. Nomura, G. N. Stemmermann, P. H. Chyou, I. Kato, G. I. Perez-Perez, and M. J. Blaser, "Helicobacter pylori infection and gastric carcinoma among Japanese Americans in Hawaii," The New England Journal of Medicine, vol. 325, no. 16, pp. 1132-1136, 1991.

[30] N. Uemura, S. Okamoto, S. Yamamoto et al., "Helicobacter pylori infection and the development of gastric cancer,"
The New England Journal of Medicine, vol. 345, no. 11, pp. 784-789, 2001.

[31] T. Kamada, J. Hata, K. Sugiu et al., "Clinical features of gastric cancer discovered after successful eradication of Helicobacter pylori: results from a 9-year prospective follow-up study in Japan," Alimentary Pharmacology \& Therapeutics, vol. 21, no. 9, pp. 1121-1126, 2005.

[32] A. Saka, K. Yagi, and S. Nimura, "Endoscopic and histological features of gastric cancers after successful Helicobacter pylori eradication therapy," Gastric Cancer, vol. 19, no. 2, pp. 524-530, 2016.

[33] K. Yagi, A. Saka, Y. Nozawa, and S. Nimura, "Endoscopic technique for qualitative diagnosis of gastric cancer and diagnosis of its expansion after successful Helicobacter pylori eradication therapy: focused on diagnosis by NBI magnifying endoscopy," Gastroenterological Endoscopy, vol. 57, no. 4, pp. 1210-1218, 2015.

[34] M. Kobayashi, S. Hashimoto, K. Nishikura et al., "Magnifying narrow-band imaging of surface maturation in early differentiated-type gastric cancers after Helicobacter pylori eradication," Journal of Gastroenterology, vol. 48, no. 12, pp. 1332-1342, 2013.

[35] M. Ito, S. Tanaka, S. Takata et al., "Morphological changes in human gastric tumours after eradication therapy of Helicobacter pylori in a short-term follow-up," Alimentary Pharmacology \& Therapeutics, vol. 21, no. 5, pp. 559-566, 2005.

[36] Y. Kitamura, M. Ito, T. Matsuo et al., "Characteristic epithelium with low-grade atypia appears on the surface of gastric cancer after successful Helicobacter pylori eradication therapy," Helicobacter, vol. 19, no. 4, pp. 289-295, 2014. 


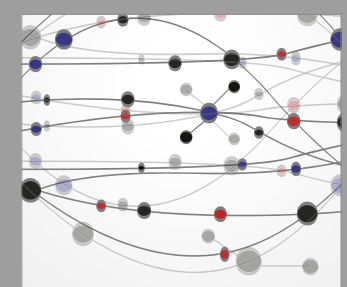

The Scientific World Journal
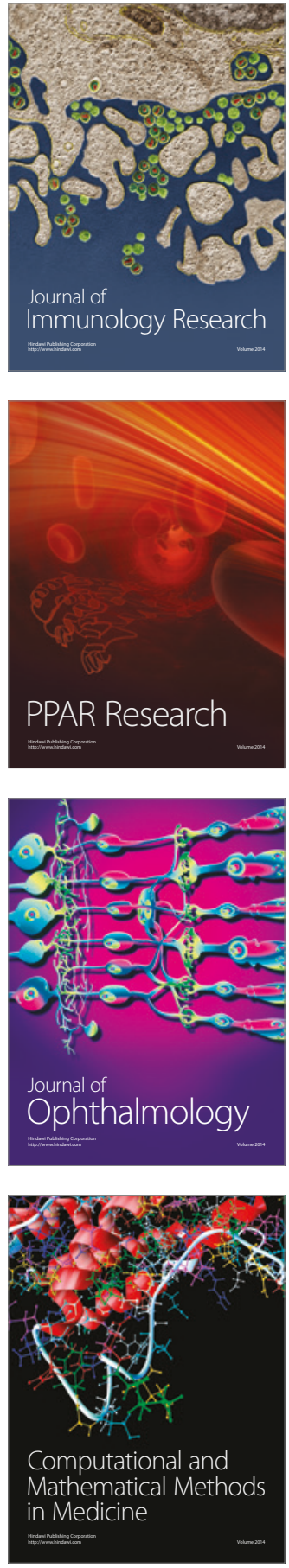

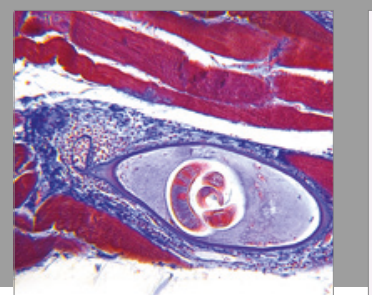

Gastroenterology Research and Practice
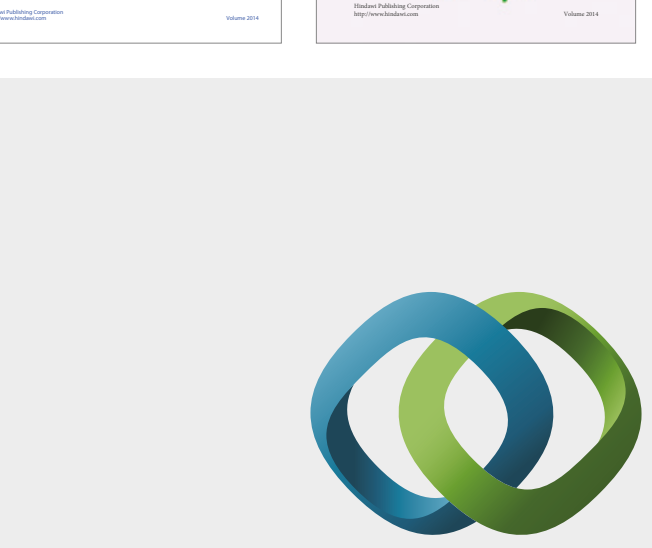

\section{Hindawi}

Submit your manuscripts at

https://www.hindawi.com
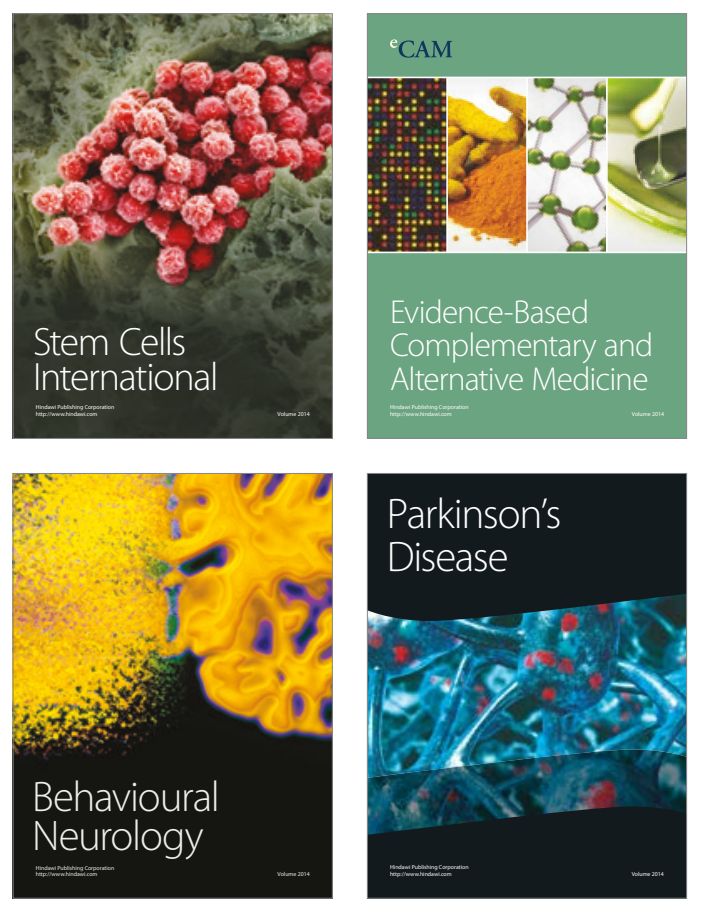
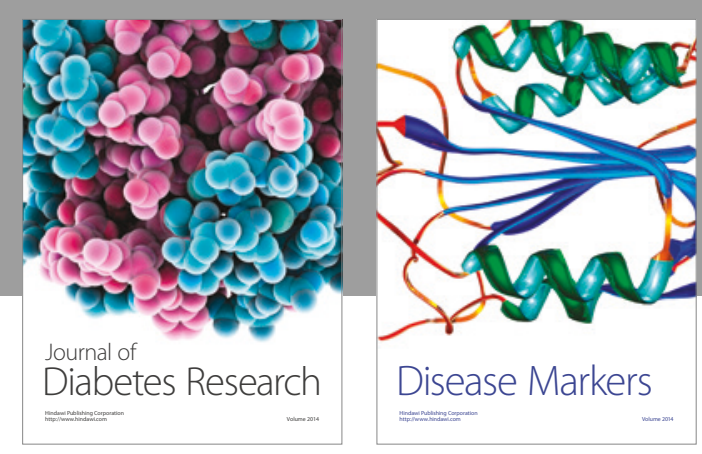

Disease Markers
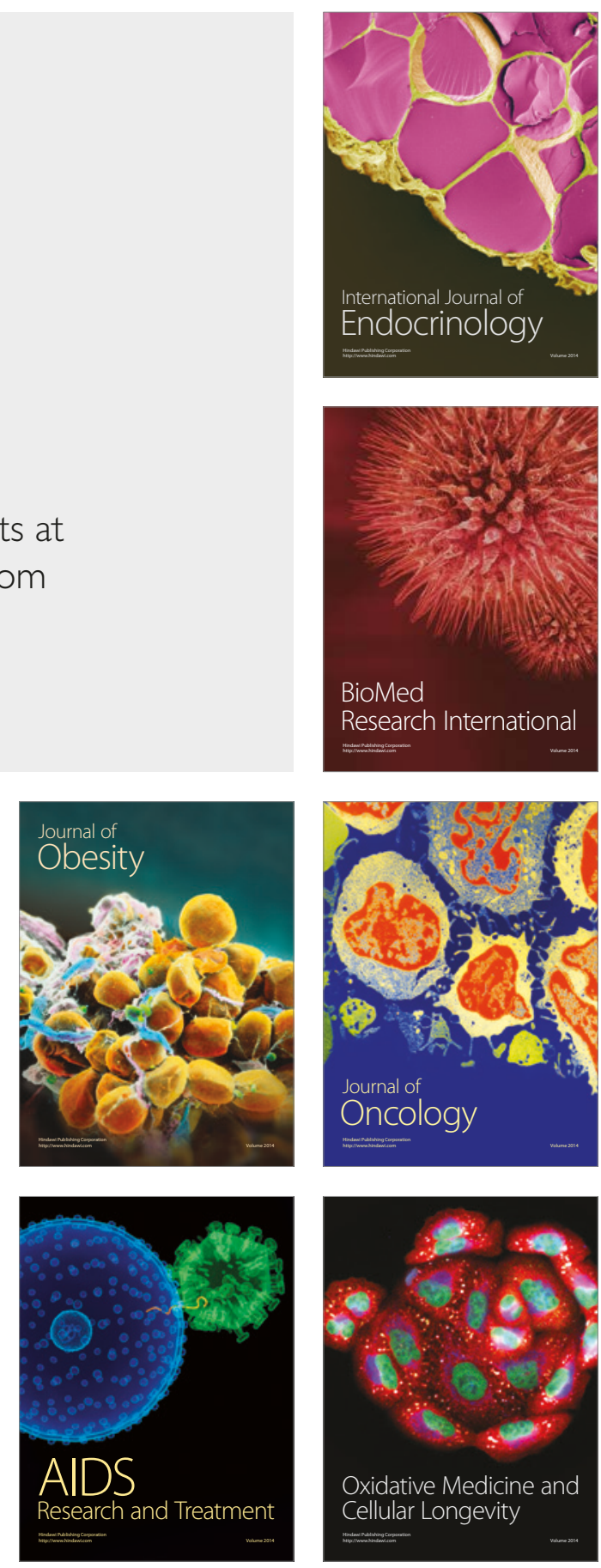